BLS 32, No 1 2006. DOI: http://dx.doi.org/10.3765/bls.v32i1.3465 (published by the Berkeley Linguistics Society and the Linguistic Society of America)

\title{
Prosody and Utterance Boundaries in American Sign Language Interpretation
}

\author{
BRENDA NICODEMUS and CAROLINE L. SMITH \\ University of New Mexico
}

\section{Introduction}

Two principal functions of prosody in spoken language are to mark prominence and to group words into larger units, or phrases. There are a variety of proposals as to the organizational structure of phrasal units, which constitute the higher levels of the prosodic hierarchy (see Shattuck-Hufnagel and Turk 1996 for a survey). Although it is clear that languages differ as to the nature and number of phrasal units that they exploit, some kind of phrasing has been identified in virtually every language that has been examined from this perspective (for an exception, see Woodbury 1993). Since phrasing is found so widely in spoken languages, the use of phrasing in signed languages should come as no surprise (Bahan and Supalla 1995, Mather and Winston 1995, Sandler 1999, Wilbur 1999). The variation observed among spoken languages suggests that certain aspects of the structure of the prosodic hierarchy, and the acoustic dimensions that reflect this structure, are language-specific. Signed languages might thus be expected to differ from spoken languages with respect to phrasal prosody because they are distinct languages with their own structural characteristics, in addition to the differences that can be explained by virtue of the distinct modality in which signed languages are expressed.

In spoken language, phrasal structure is used by speakers to organize the message they are communicating, and by perceivers to process the input (Cutler, Dahan and van Donselaar 1997). The principal acoustic dimensions that are involved are f0, duration, intensity and segmental spectral properties. The dimensions used to mark phrasing in signed languages are less well-studied, but may include variation in duration and rhythm (Boyes Braem 1999), nonmanual articulations (Wilbur 2000), and use of the nondominant hand (Sandler 2006).

The present study differs from many others in examining prosody as produced by interpreters, rather than native users of signed language. Relatively few studies have looked at interpreters' use of prosody, although one recent study focused on prosodic features that mark topic boundaries in both interpretation and translitera- 


\section{Brenda Nicodemus and Caroline L. Smith}

tion (Winston and Monikowski 2004). This issue is important because interpreters need to use prosody effectively to indicate boundaries in order to create interpretations that may be more readily processed by Deaf consumers. This task is especially salient for the interpreting profession since most interpreters are second language learners of American Sign Language (ASL) and do not have native competency with the language.

The goal of the study reported here was to examine the type, frequency, and occurrence of prosodic markers that are identified by Deaf consumers of interpreting services. The markers will be examined so as to better determine the form and function of the prosodic boundary markers used in ASL interpretation.

Defining sentence structure in signed languages is an ongoing endeavor (EngbergPedersen 2006, Hansen and Hessmann 2006) and this paper makes no claims that the identified boundaries are indicating syntactically-defined sentences, nor is it claimed that these boundaries correspond to sentence structure in spoken languages. This study does suggest, however, that Deaf participants can identify some type of boundaries in ASL interpretation and that these boundaries segment the stream of language to facilitate comprehension.

\section{Research Methodology}

This project was designed to examine the prosodic markers used to indicate boundaries during an ASL interpretation. Research has shown that listeners are sensitive to the markers that cue boundaries in spoken discourse. These boundaries serve to segment the discourse into meaningful units. In order to identify the boundaries that are meaningful to the consumers of interpreted discourse, Deaf people were asked to provide their perceptions of sentence boundaries in an ASL interpretation. This section describes the design of the stimulus material for the study and the Deaf people who served as participants in the project.

\subsection{Creation of a Source Language Text}

The first step of the research project was to create a segment of spoken English discourse to serve as the source language text for the interpreters. It was decided that a lecture would be an ideal language sample since lectures are typically monologic and a frequently interpreted type of discourse. A professor from the University of New Mexico agreed to provide a 15-minute lecture on a topic of his choosing. He selected the topic, "The Life of the Ant" which covered various aspects of ant life, including their eating and mating practices. The professor was videotaped on a Sony TRV38 Digital Camcorder (mini-DV format) as he presented the lecture to an audience of three graduate students.

\subsection{Creation of a Target Language Text}

The second step of the research project was to have the spoken English lecture interpreted into the target language, American Sign Language. Five signed language interpreters were recruited to provide an ASL interpretation of the lecture. All five interpreters were certified by the national interpreter certifying 


\section{Prosody and Utterance Boundaries in ASL Interpretation}

association. Four of the interpreters were female and one was male. One interpreter acquired ASL natively from her Deaf parents. The other four learned ASL through interpreter education programs and contact with members of the Deaf community. The years of professional experience of the interpreters ranged from 7 to 25 years.

All five interpreters provided their interpretation of the lecture individually under the same conditions. Upon arrival at the videotaping site, the interpreters were told that they would be interpreting two videotaped segments from spoken English into ASL. The two segments were described as a 3-minute introduction of the presenter and a 15-minute lecture entitled "The Life of the Ant".

The interpreters were asked to sign a consent form and complete a professional history summary. They were given preparation materials that are typical of those provided at an interpreting assignment, i.e., an outline of the lecture and a list of key terms. In addition the interpreters viewed a brief videotape of the professor giving an overview of the lecture content.

The interpreters were not informed of the specific focus of the research project. They were only told that a particular linguistic feature of their interpretation would be analyzed. When they were ready to begin, the interpreters were videotaped as they provided an ASL interpretation for both the introduction and the 15-minute lecture. A Deaf native user of ASL served as the audience for all five interpretations. The interpreters were videotaped using the Sony TRV38 Digital Camcorder (mini-DV format).

\subsection{Creation of the Stimulus Video}

The third step of the research project was to create the stimulus video for viewing by the Deaf participants. The stimulus video was composed of three sections: (1) the directions, (2) the practice trial, and (3) the interpretation.

\subsubsection{Directions}

The first portion of the stimulus material was the directions for the research study. The directions were provided in American Sign Language by a Deaf, native user of ASL. The directions stated that participants were participating in a research project about how interpreters created sentence boundaries in ASL interpretation. The participants were asked to look for the completion of a full idea, concept or "sentence." The term "sentence" was used because of its familiarity. The participants were directed to view a videotaped ASL interpretation on a laptop and press an attached button each time they perceived a sentence boundary. The directions also stated that participants would be given a short practice trial prior to performing the actual research task.

\subsubsection{Practice Trial}

The second section of the stimulus material was a practice trial. The practice trial consisted of a three-minute ASL interpretation of a speaker introducing the presenter. During this trial, the Deaf participants practiced pressing the button at 


\section{Brenda Nicodemus and Caroline L. Smith}

perceived sentence boundaries in the interpretation. They were given the opportunity to perform the practice trial as many times as they wished.

\subsubsection{Interpretation}

The third section of the stimulus material was the videotaped ASL interpretation of the 15-minute lecture. The lecture was divided into three-minute segments with a 15-second break between them to reduce the risk of fatigue for the Deaf participants.

\subsubsection{Presentation of the Stimulus Material}

Each of the three sections of the stimulus material was videotaped in digital format and transferred into iMovie software. The files were edited and saved on a Macintosh PowerBook G4 which was also used to present the stimulus material to the Deaf participants. When indicating the perception of a boundary, the participants pressed a Powermate button which left a visual indicator in the iMovie software. The total length of the stimulus material videotape was 22 minutes.

\subsection{Deaf Participants}

The role of the Deaf participants was to evaluate the occurrence of sentence boundaries in an ASL interpretation. In this section, the Deaf participants are described along with the setting and procedures used in research process.

Fifty Deaf participants took part in this research project. The participants had to satisfy the following criteria:

a) Identify as a member of the Deaf community;

b) Report that ASL was their primary language;

c) Be 18 years of age or older; and

d) Report frequent use of signed language interpreters.

The participants resided in 10 different states and the District of Columbia. They were fairly evenly divided across gender (males $=21$, females $=29$ ) and represented a broad spectrum of age ranges and levels of education. The majority of the participants were employed at residential schools for the deaf.

The participants responded to a call for subjects at either a residential school or a conference. They were recruited in geographical areas other than where the interpreters work in order to assure anonymity and eliminate potential bias from prior experience with the interpreters. They participated voluntarily and were paid twenty dollars apiece for their involvement. Prior to participating in the study, each participant was asked to sign a consent form and complete a personal information sheet.

The participants were told that they were engaging in a linguistic research project on signed language interpreters. They viewed the directions for the project and were allowed the opportunity for questions. They then performed the practice trial task. When the participant indicated readiness to begin the study, the videotape was started and the researcher left the immediate environment. 


\section{Prosody and Utterance Boundaries in ASL Interpretation}

As stated earlier, five interpreters were videotaped providing separate interpretations of the lecture. It should be noted that each of the Deaf participants viewed only one of the interpreters. Ten Deaf participants viewed the first interpreter, another ten viewed the second interpreter, and so on, for a total of 50 Deaf participants watching five separate interpreters. The study was designed in this way in order to examine the variety of markers that were employed by the individual interpreters.

\section{Data Analysis}

The section of the paper describes the procedures for collecting and analyzing the data including defining "clusters of agreement" and the type of prosodic markers identified in the coding.

\subsection{Defining "Clusters of Agreement"}

Ten Deaf participants viewed a single interpretation and responded to perceived sentence boundaries by pressing a button. Analysis of the participants' responses focused on "clusters of agreement" which were defined as intervals where six or more of the Deaf participants (out of ten) responded within one second. This onesecond interval of agreement was also employed in an examination of prosodic markers in British Sign Language (Fenlon, Denmark and Woll 2006).

Across the five interpretations, there was a total of 153 "clusters of agreement" on the location of sentence boundaries. The number of "clusters of agreement" varied among the interpreters, as shown in Table (1).

Number of clusters of agreement identified in each interpretation

\begin{tabular}{|c|c|c|c|c|c|}
\hline \multicolumn{5}{|c|}{ Interpreters } & Total \\
\hline A & B & C & D & E & \\
\hline 46 & 6 & 36 & 21 & 44 & 153 \\
\hline
\end{tabular}

The variation in number of agreement points among the interpreters may be due to several factors, including varying attention by participants, or the idiosyncratic use of prosody by the interpreters that may not have consistently cued the participants. It is also possible that the methodology of this study did not fully capture the variation in the interpreters' use of prosodic markers. It should be noted that in informal interviews following the study, all Deaf participants said that they felt the interpreters were successful in conveying the information in the lecture.

These 153 agreement points were time-aligned to the digitized interpretations for analysis. The analysis of the interpreters' production of prosodic markers examined a two-second interval, beginning one second prior to the first participant's response, and ending at the final response in the cluster. The two-second interval in the interpretation was analyzed and coded for the type of prosodic markers that occurred, the frequency of the markers, and the number of overlapping markers. 


\section{Brenda Nicodemus and Caroline L. Smith}

\subsection{Coding the Data}

The prosodic markers were analyzed and coded by identifying specific physical movements of the interpreter. The range of prosodic markers produced in signed languages have been described by various researchers and served as a foundation for this analysis. A total of 21 prosodic markers were coded for in the analysis. The markers were coded only if there was a change in state when they were produced. In other words, if a marker, such as a body lean, was held constant throughout the two second interval, it was not coded as marking the sentence boundary.

The videotaped interpretations were viewed frame by frame and coded across each prosodic marker type. Codes were used to designate the specific shift in movement, and each prosodic marker was coded for its duration.

The prosodic markers were designated as falling into one of four broad categories based on specific articulators used in signed languages. The four categories were:

Category 1 - Hands

Category 2 - Head and Neck

Category 3 - Eyes, Nose, and Mouth

Category 4 - Body

A description of the specific prosodic markers for each category follows.

\section{Category 1 - Hands}

Coding in Category 1 occurred across the following five prosodic markers.

1. Held Handshape - Describes the holding or "freezing" of a handshape.

2. Hand Clasp - Describes the behavior in which the interpreter clasps his/her hands in front of the body. The fingers may be interlocked or folded. The hand clasp tends to occur around the waist with the elbows at a 90 degree angle.

3. Fingers Wiggling - Describes the behavior when one (or both) hands are being suspended in a neutral space in front of the interpreter's body and the fingers are wiggling.

4. Hands Drop - Describes the behavior of one (or both) hands having completed a sign and are dropped and held without movement.

5. Signing Space - Describes the behavior in which the hands are signing to the left or right of the interpreter's body.

\section{Category 2 - Head and Neck}

In this category the head is described by movements that involve rotation with respect to an $\mathrm{X}, \mathrm{Y}$, and $\mathrm{Z}$ axis. For all head position fields, the head first moves to a target position and then maintains that position as some value (front, back, left right, etc.).

Coding in Category 2 occurred across the following seven prosodic markers.

1. Head Position: Tilt (Front and Back) - In this position the interpreter's head is tilted forward or backwards and maintains that position for some length of time. 


\section{Prosody and Utterance Boundaries in ASL Interpretation}

2. Head Position: Turn (Left and Right) - In this position, the interpreter's head has rotated on the $\mathrm{Y}$ axis (which may be envisioned as a vertical rod originating at the middle of the skull). The head is turned either left or right and held in that position.

3. Head Position: Tilt (Left and Right) - In this position, the interpreter's head has rotated on the $\mathrm{Z}$ axis (which may be envisioned as a horizontal rod originating at the back of the skull and coming through the nose). The head is tilted either left or right and held in that position for some length of time.

4. Head Movement: Nod - In this motion, the interpreter's head is moving on the $\mathrm{X}$ axis which may be envisioned as a rod running from ear to ear). The head is moving up and down in this position in a repetitive motion.

5. Head Movement: Shake - In this motion, the interpreter's head is moving on the $\mathrm{Y}$ axis (which may be envisioned as a vertical rod originating at the middle of the skull). The head is moving left and right and held in a repetitive motion.

6. Head Movement: Side to Side - In this motion, the interpreter's head is moving on the $\mathrm{Z}$ axis (which may be envisioned as a horizontal rod originating at the back of the skull and coming through the nose). The head is moving left and right in a repetitive manner.

7. Neck - Describes a shift in the muscular tension of the neck.

\section{Category 3 - Eye, Nose and Mouth}

Coding in Category 3 occurred across the following five prosodic markers.

1. Eyebrows - Denotes a shift in the interpreter's eyebrow height.

2. Eye Gaze - Denotes a shift the direction of the interpreter's eye gaze.

3. Eye Aperture - Denotes a shift in the degree of the eyelid movement such as squinting, widening, or closing.

4. Nose - Denotes that the interpreter is "wrinkling" his/her nose.

5. Cheeks - Denotes a shift in the cheeks by puffing or releasing a puff.

\section{Category 4 - Body}

Coding in Category 4 occurred across the following four prosodic markers.

1. Body Lean - Denotes a shift and hold of the direction of the interpreter's body.

2. Body Movement - Denotes movement of the interpreter's body that continues in a repetitive manner.

3. Large Breath - Denotes the presence of a visible breath.

4. Shoulders - Denotes that the behavior of the shoulders being raised or lowered.

\section{Results}

The initial analysis resulted in two findings: (1) the frequency of prosodic marker type, and (2) the number of prosodic markers at the identified sentence boundaries. 
Brenda Nicodemus and Caroline L. Smith

\subsection{Frequency of Prosodic Marker Type}

Coding the results revealed the most frequent prosodic marker type in each of the four articulator categories. The hands (Category 1) and the body (Category 4) each had a single marker that was dominant, while the head and neck (Category 2 ) and the eyes, nose and mouth (Category 3 ) used several different markers. This difference suggests that the fine motor movements of each of the individual articulators can act independently of one another. In addition, the results show that, although the interpreters show variation in their production of prosodic markers, there are specific markers that fairly consistently occur more frequently than others.

(2) Frequency of Markers in Category 1 - Hands

\begin{tabular}{|l|c|c|c|c|c|c|}
\hline & A & B & C & D & E & Total \\
\hline Hand Clasp & 42 & 3 & 28 & 8 & 19 & 100 \\
\hline Held Handshape & 16 & 2 & 10 & 4 & 6 & 38 \\
\hline Sign Space & 6 & 3 & 6 & 2 & 6 & 23 \\
\hline Hands Drop & 1 & 0 & 3 & 3 & 11 & 18 \\
\hline Fingers Wiggling & 0 & 0 & 0 & 0 & 0 & 0 \\
\hline
\end{tabular}

(3) Frequency of Markers in Category $2-$ Head and Neck

\begin{tabular}{|l|c|c|c|c|c|c|}
\hline & A & B & C & D & E & Total \\
\hline Head Position: Tilt (F/B) & 32 & 3 & 19 & 15 & 26 & 95 \\
\hline Head Position: Turn (L/R) & 31 & 1 & 16 & 7 & 19 & 74 \\
\hline Head Movement: Nod & 14 & 0 & 16 & 7 & 30 & 67 \\
\hline Head Position: Tilt (L/R) & 6 & 1 & 18 & 10 & 12 & 47 \\
\hline Head Movement: Shake & 2 & 0 & 2 & 3 & 2 & 9 \\
\hline Head Movement: Side to Side & 0 & 1 & 0 & 0 & 1 & 2 \\
\hline Neck & 0 & 0 & 0 & 0 & 0 & 0 \\
\hline
\end{tabular}

(4) Frequency of Markers in Category 3 - Eyes, Nose, and Mouth

\begin{tabular}{|l|c|c|c|c|c|c|}
\hline & A & B & C & D & E & Total \\
\hline Eye Aperture & 43 & 6 & 35 & 17 & 44 & 145 \\
\hline Eyebrows & 32 & 4 & 29 & 12 & 42 & 119 \\
\hline Eye Gaze & 30 & 6 & 27 & 13 & 42 & 118 \\
\hline Nose Wrinkling & 0 & 0 & 4 & 0 & 0 & 4 \\
\hline Puffed Cheeks & 1 & 0 & 0 & 0 & 2 & 3 \\
\hline
\end{tabular}

(5) Frequency of Markers in Category 4 - Body

\begin{tabular}{|l|c|c|c|c|c|c|}
\hline & A & B & C & D & E & Total \\
\hline Body Lean & 41 & 3 & 23 & 7 & 22 & 96 \\
\hline Shoulders & 9 & 0 & 10 & 3 & 2 & 24 \\
\hline Body Movement & 8 & 1 & 2 & 1 & 0 & 12 \\
\hline Breath & 0 & 0 & 0 & 0 & 0 & 0 \\
\hline
\end{tabular}




\section{Prosody and Utterance Boundaries in ASL Interpretation}

\subsection{Number of Prosodic Markers at Boundaries}

A second question that was investigated is the number of prosodic markers employed at the boundaries. American Sign Language has been described as being "layered" (Wilbur 2000) because it enables the use of multiple articulators simultaneously. Analysis of the data reveals that prosodic cues that mark boundaries are also produced in a layered manner. Table (6) outlines the number of markers that were observed in the two-second interval of interpretation. The chart shows that the number of prosodic markers being produced most often falls between five and eight per interval.

(6)

Number of Prosodic Markers

\begin{tabular}{|l|c|c|c|c|c|c|}
\hline & A & B & C & D & E & Total \\
\hline Three Markers & 3 & 0 & 0 & 3 & 0 & 6 \\
\hline Four Markers & 0 & 1 & 1 & 2 & 0 & 4 \\
\hline Five Markers & 5 & 2 & 2 & 6 & 8 & 23 \\
\hline Six Markers & 6 & 1 & 13 & 5 & 13 & 38 \\
\hline Seven Markers & 16 & 0 & 9 & 4 & 14 & 43 \\
\hline Eight Markers & 10 & 2 & 6 & 1 & 5 & 24 \\
\hline Nine Markers & 6 & 0 & 5 & 0 & 3 & 14 \\
\hline Ten Markers & 0 & 0 & 0 & 0 & 1 & 1 \\
\hline TOTAL & 46 & 6 & 36 & 21 & 44 & 153 \\
\hline
\end{tabular}

\section{Discussion and Conclusions}

The initial analysis of the type, frequency and number of prosodic markers employed in ASL interpretation suggests that, as with spoken language, people have the ability to detect when prosodic markers are being used for lexical/grammatical purposes versus when they are used for marking phrasal boundaries. One of the surprising findings of this study was the high number of markers used by interpreters during a very short interval of time. Although the number of markers used is relatively high, the data suggest that there is a limited set of prosodic markers used by interpreters to indicate boundaries and that their usage demonstrates a range of stylistic variation is possible while still producing a successful interpretation.

It was observed that when multiple prosodic markers occur together in ASL interpretation (which is almost always the case) they can occur either simultaneously or sequentially. One hypothesis is that the sequential production may be used because of the physical constraints of a visual-gestural language modality. For example, if one large articulator (e.g., body lean, hand clasp, shoulder raise) is being employed to mark a boundary, no other large articulator can occur simultaneously. If a second large articulator is used to mark the boundary, it occurs in a sequential manner. However, fine motor movements (eye aperture, head tilt, head nod) can be used simultaneously with a large articulator. The combination of the more fine-grained articulations with the larger articulations may serve to reinforce 
Brenda Nicodemus and Caroline L. Smith

the presence of a boundary. Further investigation will reveal how these articulator categories are timed and coordinated to effectively cue boundaries.

Deeper analysis of the timing and duration of the markers and their sequencing in combination is needed. It would also be enlightening to do a parallel examination of the use of prosodic markers by native users of ASL. The findings from this study will add to the body of knowledge of how human languages employ prosodic structure. In the end, the similarities between the functional role of prosody in spoken and signed languages appear to be more striking than the differences.

\section{References}

Bahan, Ben, and Sam Supalla. 1995. Line Segmentation and Narrative Structure. In K. Emmorey and J. Reilly, eds., Language, Gesture, and Space, 171-191. Hillsdale, NJ: Lawrence Erlbaum Associates Publishers.

Boyes Braem, Penny. 1999. Rhythmic Temporal Patterns in the Signing of Deaf Early and Late Learners of Swiss German Sign Language. Language and Speech 42(2/3):177-208.

Cutler, Anne, Delphine Dahan, and Wilma van Donselaar. 1997. Prosody in the Comprehension of Spoken Language: A Literature Review. Language and Speech 40(2):141-201.

Engberg-Pederson, Elisabeth. 2006. Intertwined Ellipsis: A Multiclausal Construction in Danish Sign Language. Paper presented at the Annual Meeting of the German Linguistics Society.

Fenlon, Jordan, Denmark, Tanya, and Bencie Woll. 2006. Seeing Sentence Boundaries. Paper presented at the Annual Meeting of the German Linguistics Society.

Hansen, Martje, and Jens Hessmann. 2006. Reanalysing Sentences in German Sign Language (DGS) Texts. Paper presented at the Annual Meeting of the German Linguistics Society.

Mather, Sue, and Elizabeth Winston. 1995. Spatial Mapping and Involvement in ASL Storytelling. In C. Lucas, ed., Pinky Extension and Eye Gaze, 183-210. Washington, DC: Gallaudet University Press.

Sandler, Wendy. 1999. Prosody in Two Natural Language Modalities. Language and Speech 42(2/3): 127-142.

Sandler, Wendy. 2006. Phonology, Phonetics and the Nondominant Hand. In L. Goldstein, D. Whalen, and C. Best, eds., Laboratory Phonology 8. Berlin: Mouton de Gruyter. 185-212.

Shattuck-Hufnagel, Stephanie, and Alice Turk. 1996. A Prosody Tutorial for Investigators of Auditory Sentence Processing. Journal of Psycholinguistic Research 25(2):193-247.

Wilbur, Ronnie. 1999. Stress in ASL: Empirical Evidence and Linguistic Issues. In Language and Speech 42(2/3):229-250. 
Wilbur, Ronnie. 2000. Phonological and Prosodic Layering of Nonmanuals in American Sign Language. In K. Emmorey and J. Lane, eds., The Signs of Language Revisited, 215-244. Mahwah, NJ: Erlbaum.

Winston, Elizabeth A., and Christine Monikowski. 2004. Marking Topic Boundaries in Signed Interpretation and Transliteration. In M. Metzer, S. Collins, V. Dively, and R. Shaw, eds., From Topic Boundaries to Omission: New Research on Interpretation, 187-227. Washington, DC: Gallaudet University Press.

Woodbury, Anthony. 1993. Against Intonational Phrases in Central Alaskan Yupik Eskimo. Paper presented at the Annual Meeting of the Linguistic Society of America.

Brenda Nicodemus

Department of Interpretation

Gallaudet University

800 Florida Avenue, NE

Washington, DC 20002

nicodemusb@gmail.com

Caroline L. Smith

Department of Linguistics

University of New Mexico

MSC 03-2130

Albuquerque, NM 87131-0001

caroline@unm.edu 